\section{Failure of Decolonization in Patients With Infections Due to Mupirocin-Resistant Strains of Community-Associated Methicillin-Resistant Staphylococcus aureus}

To the Editor-We read the recent article by Rahimian et al. ${ }^{1}$ with great interest, and we applaud the authors for their efforts in addressing the role of treatment with mupirocin for recurrent methicillin-resistant Staphylococcus aureus (MRSA) skin and skin structure infections. We hypothesize that one potential reason for the high rate of recurrence of skin and skin structure infections observed by Rahimian et al. ${ }^{1}$ in patients with MRSA nasal colonization treated with mupirocin (6 [32\%] of 19) may be plasmid-mediated resistance to mupirocin. In a prior publication, Shastry et al. ${ }^{2}$ demonstrated that there was a very high level of clindamycin resistance in their population of men who have sex with men (63 [63\%] of 100).

We have demonstrated that clindamycin resistance and mupirocin resistance are both encoded on a single plasmid, pUSA03, that is frequently identified in multidrug-resistant strains of community-associated MRSA genotype USA $300 .^{3}$ We have noted that the pUSA03-positive USA300 subclone is particularly prevalent as a cause of skin and skin structure infections in the population of men who have sex with men in San Francisco and Boston. ${ }^{4}$ Most notably, this subclone was the pathogen in skin and skin structure infections in men who have sex with men who had no history of prior clindamycin or mupirocin use, suggesting person-to-person transmission of the multidrug-resistant USA300 clone.

With respect to the study by Rahimian et al., ${ }^{1}$ it would be of great interest to know (1) how many of the 19 patients treated with mupirocin had initial infecting and nasal colonizing strains resistant to both clindamycin and mupirocin (thus suggesting the presence of pUSA03) and (2) how many of these patients were men who have sex with men. Although the findings of Rahimian et al. ${ }^{1}$ indicate that an attempt at decolonization with mupirocin may not be beneficial in preventing recurrent disease due to community-associated MRSA in their patient population, the effect of decolonization with mupirocin in a population with lower rates of clindamycin and mupirocin resistance in colonizing and/or infecting MRSA strains remains undetermined.

\section{ACKNOWLEDGMENTS}

Potential conflicts of interest. The authors report no conflicts of interest relevant to this article.

Christopher J. Graber, MD, MPH; Brian S. Schwartz, MD
From the Department of Medicine, Division of Infectious Diseases, San Francisco General Hospital, University of California, San Francisco (both authors).

Address reprint requests to Christopher Graber, MD, MPH, Department of Medicine, Division of Infectious Diseases, San Francisco General Hospital, University of California, San Francisco, 1001 Potrero Avenue, Building 30, Box 0811, San Francisco, CA 94110 (graber_chris@hotmail.com).

Infect Control Hosp Epidemiol 2008; 29:284-284

(C) 2008 by The Society for Healthcare Epidemiology of America. All rights reserved. 0899-823X/2008/2903-0015\$15.00. DOI: 10.1086/527451

\section{REFERENCES}

1. Rahimian J, Khan R, LaScalea KA. Does nasal colonization or mupirocin treatment affect recurrence of methicillin-resistant Staphylococcus aureus skin and skin structure infections? Infect Control Hosp Epidemiol 2007; 28:1415-1416.

2. Shastry L, Rahimian J, Lascher S. Community-associated methicillin-resistant Staphylococcus aureus skin and soft tissue infections in men who have sex with men in New York City. Arch Intern Med 2007; 167:854-857.

3. Diep BA, Gill SR, Chang RF, et al. Complete genome sequence of USA300, an epidemic clone of community-acquired meticillin-resistant Staphylococcus aureus. Lancet 2006; 367:731-739.

4. Diep BA, Chambers HF, Graber CJ, et al. Emergence of multidrug resistant community-acquired methicillin resistant Staphylococcus aureus clone USA300 in men who have sex with men (MSM). In: Proceedings of the 47th Interscience Conference on Antimicrobial Agents and Chemotherapy. Washington, DC: American Society for Microbiology; 2007:109. Abstract C2-178.

\section{Reply to Graber and Schwartz}

To the Editor-We appreciate the insightful comments by Graber and Schwartz ${ }^{1}$ regarding our article on mupirocin treatment for recurrence of community-associated methicillin-resistant Staphylococcus aureus (CA-MRSA) skin and skin structure infections. ${ }^{2}$ The high number of recurrences of colonization that we found in patients treated with mupirocin may indeed be unique to our study population. Among a subset of our study population (ie, 19 patients who had nasal MRSA colonization that was treated with mupirocin), 17 were men who have sex with men, a population that we previously found to have a high rate of colonization with clindamycinresistant CA-MRSA strains. ${ }^{3}$ Of the 19 colonized patients treated with mupirocin, 15 carried MRSA strains in their nares that were resistant to clindamycin (of note, all 15 of these patients were men who have sex with men).

Unfortunately, our laboratory did not test strains for mupirocin susceptibility. However, given that mupirocin resistance and clindamycin resistance are both encoded on the pUSA03 plasmid, ${ }^{4}$ it is likely that a significant number of 
these patients were also had strains resistant to mupirocin and that this could potentially account for the high number of recurrences of colonization seen in our study population. Thus, it is possible that our study underestimates the potential benefit of mupirocin treatment for other patient populations colonized with CA-MRSA. Further studies are needed to help clarify the potential benefit of mupirocin treatment for nasal decolonization among patients with CA-MRSA nasal carriage.

\section{ACKNOWLEDGMENTS}

Potential conflicts of interest. The author reports no conflicts of interest relevant to this article.

Joseph Rahimian, MD

From New York Medical College, St. Vincent Hospital, New York, New York. Address reprint requests to Joseph Rahimian, MD, New York Medical College, St. Vincent Hospital, 153 W. 11th Street, New York, NY 10011 (villagemd@yahoo.com).

Infect Control Hosp Epidemiol 2008; 29:284-285

(C) 2008 by The Society for Healthcare Epidemiology of America. All rights reserved. 0899-823X/2008/2903-0016\$15.00.DOI: $10.1086 / 527452$

\section{REFERENCES}

1. Graber CJ, Schwartz BS. Failure of decolonization in patients with infections due to mupirocin-resistant strains of community-associated methicillin-resistant Staphylococcus aureus. Infect Control Hosp Epidemiol 2008; 29:284 (in this issue).

2. Rahimian J, Khan R, LaScalea KA. Does nasal colonization or mupirocin treatment affect recurrence of methicillin-resistant Staphylococcus aureus skin and skin structure infections? Infect Control Hosp Epidemiol 2007; 28:1415-1416.

3. Shastry L, Rahimian J, Lascher S. Community-associated methicillin-resistant Staphylococcus aureus skin and soft tissue infections in men who have sex with men in New York City. Arch Intern Med 2007; 167:854-857.

4. Diep BA, Gill SR, Chang RF, et al. Complete genome sequence of USA300, an epidemic clone of community-acquired methicillin-resistant Staphylococcus aureus. Lancet 2006; 367:731-739.

\section{Intervention to Increase Influenza Vaccination Rates Among Healthcare Workers in a Tertiary Teaching Hospital in Brazil}

TO THE EDITOR-Annual influenza vaccination is recommended for persons at higher risk for severe disease and complications related to influenza and for persons who live with or care for those at higher risk. ${ }^{1,2}$ The goal of an influenza vaccination program in a healthcare facility is to prevent transmission of the virus and the illness among those at high risk. ${ }^{1,2}$ Healthcare workers (HCWs) often continue to work when infected with influenza. In a survey of employees of the Hospital das Clinicas, in Sao Paulo, Southeast of Brazil, $94 \%$ of the HCWs stated that they had come to work with influenza-like illness. ${ }^{3}$ Vaccination of HCWs is associated with a reduction in deaths from pneumonia and deaths from all causes among nursing home patients. ${ }^{4}$ Occupational health vaccination programs have also been shown to reduce staff illness and absenteeism, prevent workplace disruption, and result in financial savings to sponsoring health institutions. ${ }^{1,5}$

Despite the recommendations, the influenza vaccination rates among HCWs remain low. ${ }^{1,6}$ Barriers to vaccine access and misconceptions regarding influenza and the vaccine have been associated with nonvaccination among HCWs. ${ }^{3,6,7}$ Interventions to enhance access to vaccination to make it more convenient for the target population have been proposed as a strategy to increase influenza vaccination rates among HCWs. $^{7}$

In January 2006, a Working Group was constituted at the Hospital das Clinicas to establish a plan of action to address the risk of pandemic influenza. Enhancing the rate of vaccination against seasonal influenza among HCWs was one of the goals proposed by this group.

Hospital das Clinicas is a 2,200-bed tertiary teaching hospital complex ( 5 buildings and 2 auxiliary hospitals) attached to the University of Sao Paulo School of Medicine. Including permanent and casual staff, employees, students, and volunteers, Hospital das Clinicas has an estimated 20,000 HCWs. Since 1999, annual influenza vaccination has been offered free of charge to all HCWs at the hospital's Immunization Center during working hours. Under this strategy, 1,202 HCWs (6\% of the target population) were vaccinated in 2004 , and $1,292(6.5 \%)$ were vaccinated in 2005 .

The strategy implemented during the 2006 season featured both an educational campaign and a vaccination campaign. The educational campaign addressed influenza and emphasized the importance and safety of vaccination through lectures, informal handouts, fact sheets distributed with employees' paychecks, and posters. The vaccination campaign offered the vaccine at places of easy access during expanded hours.

The HCW vaccination campaign was conducted at the same time as the National Annual Influenza Vaccination Campaign, from April 24 to May 8, 2006. In the main building of the hospital, the vaccination was made available by mobile teams carrying coolers stocked with vaccine, a minimummaximum thermometer, ice packs, syringes, alcohol hand rub, alcohol wipes, adhesive bandages, disposal containers for needles, and documentation forms. At the change of shift, the mobile teams were located in the hall of each of the 2 main HCW entrances. The rest of the day, the mobile teams walked all the floors of the building visiting inpatient wards, the emergency department, laboratories, and the radiology department. In the other buildings of the complex, the vaccine was offered to the HCWs in places of easy access, but mobile vaccination was not implemented.

The nurses who worked in the campaign were given a 12 - 